\title{
X-Ray Absorption Characterization of Supported Palladium Catalysts: A Comparison with Hydrogen Chemisorption
}

\author{
G. Zhang, M.M. Habib and S.E. Vittoratos \\ Chevron Research and Technology Company, 100 Chevron Way, Richmond, CA 94802, U.S.A.
}

\begin{abstract}
X-ray absorption spectroscopy was used to characterize the supported palladium hydrocracking catalysts. Results with regard to the percentage of metal exposed are compared with those from hydrogen chemisorption measurements. Results from XAS indicate that hydrogen chemisorption has underestimated the metal dispersion. Possible explanations for this discrepancy are discussed.
\end{abstract}

\section{INTRODUCTION}

Supported palladium catalysts are widely used to hydrocrack heavy crude fractions to transportation fuels of higher value. Among their specifications, the percentage of metal exposed is a very important parameter in control of the catalyst manufacture and performance. This is usually determined by hydrogen chemisorption because of its low cost and convenience. However, results from conventional hydrogen chemisorption often disagree with other techniques for the supported palladium catalysts. In addition, carbon coverage of the spent catalysts can totally disable the hydrogen chemisorption method. X-ray absorption, an element-specific technology, has shown potential advantage over chemisorption in characterizing the Pd catalysts. In this paper we use XAS to characterize the supported palladium catalysts and evaluate the chemisorption method.

\section{EXPERIMENTAL}

Catalysts A and B contain a few tenths of a weight percent of Pd supported on amorphous silica-alumina with alumina as a binder. We ground the catalyst pellets to powder, pressed it into a self-supporting wafer, mounted the wafer in our controlled atmosphere catalyst sample chamber. The catalysts were dried and reduced in-situ with flowing hydrogen at $320^{\circ} \mathrm{C}$ for one hour, followed by evacuation at $320^{\circ} \mathrm{C}$ for 0.5 hour, then cooled to room temperature for data collection.

X-ray absorption at the Pd K edge was measured at Beam Line X18B at Brookhaven National Synchrotron Light Source. This is a bending magnet beam line equipped with a channel cut Si(111) monochromator. Synchrotron radiation was generated from an electron storage ring running at $2.584 \mathrm{GeV}$ with $140-300 \mathrm{~mA}$ of current. All data were collected at room temperature in transmission configuration.

X-ray absorption data were reduced and analyzed in a standard manner using the University of Washington data analysis package[1]. Contributions from individual coordination shells were separated by Fourier transforming the spectra from 5.0 to $13.0 \AA^{-1}$ using $\mathrm{k}^{3}$ weighting and a Hanning window function with $2.0 \AA^{-1}$ sills at each end. The experimental data in 1.8 $3.2 \AA$ were then fitted by means of FEFFIT with a theoretical Pd-Pd absorption function synthesized from FEFF 6.0. The amplitude reduction factor of 0.70 , which was derived from parameter fitting of the experimental Pd foil XAS data, was employed in the fitting process.

For chemisorption measurements, the catalysts were dried at increasing temperatures up to $350^{\circ} \mathrm{C}$, then reduced at $320^{\circ} \mathrm{C}$ for 2 hours, followed by evacuation at $350^{\circ} \mathrm{C}$ for 0.5 hour. Volumetric hydrogen chemisorption measurements started after the samples were cooled to $110^{\circ} \mathrm{C}$ under vacuum. Then the catalysts were evacuated again for back-sorption measurements. The hydrogen uptake measured in the back-sorption experiment is referred as reversibly chemisorbed hydrogen, while the difference between two hydrogen uptakes as irreversibly chemisorbed hydrogen.

\section{RESULTS AND DISCUSSION}

The Fourier transformed fine structures of catalysts A and B are shown in Figure 1. Results from X-ray absorption and hydrogen chemisorption measurements are compared in Table 1. 
Table 1: Comparison of Results Regarding Metal Dispersion Between XAS and Hydrogen Chemisorption

\begin{tabular}{|l|lllll|lll|}
\hline & \multicolumn{5}{|c|}{ X-Ray Absorption } & \multicolumn{2}{|c|}{ Hydrogen Chemisorption } \\
& $\mathrm{R}(\mathrm{Pd}-\mathrm{Pd}) / \AA$ & $\mathrm{CN}(\mathrm{Pd}-\mathrm{Pd})$ & Sigma $^{2} / \AA^{2}$ & Diameter & Dispersion & $\mathrm{H}(\mathrm{i}) / \mathrm{Pd}$ & $\mathrm{H}(\mathrm{r}) / \mathrm{Pd}$ & $\mathrm{H}(\mathrm{t}) / \mathrm{Pd}$ \\
Catalyst A & $2.732 \pm 0.006$ & $7.8 \pm 1.1$ & $0.0068 \pm 0.0007$ & $\approx 15 \AA$ & $75 \%$ & 0.199 & 0.219 & 0.419 \\
Catalyst B & $2.719 \pm 0.004$ & $4.3 \pm 0.4$ & $0.0109 \pm 0.0006$ & $<8 \AA$ & $100 \%$ & 0.363 & 0.322 & 0.685 \\
\hline
\end{tabular}

Note: $\quad H(i), H(r)$ and $H(t)$ refer to irreversibly, reversibly and total chemisorbed hydrogen respectively.

From the coordination number of 7.8 , we estimate that Pt particles of about $15 \AA$ on average have formed on catalyst $A$. From Figure 1, we can see that catalyst $A$ follows the Fourier pattern of Pd foil very well to more than seven coordination shells, confirming the formation of large metal particles. The small coordination number of 4.3 indicates that palladium forms small clusters of less than $10 \mathrm{Pd}$ atoms on catalyst $\mathrm{B}$. These disagree with the results from hydrogen chemisorption. Assuming the stoichiometry between hydrogen and surface $\mathrm{Pd}$ is $1: 1$, the values of metal dispersion calculated from irreversibly chemisorbed hydrogen are much lower than the estimates from X-ray absorption measurements. If the total hydrogen uptake is taken into account, the values from chemisorption are still $30-40 \%$ too low.

The above comparison clearly indicates that hydrogen chemisorption has underestimated the metal dispersion. There may be a number of possible explanations for this discrepancy. The stoichiometry of H:Pd might be less than 1; the hydrogen uptake might be particle size dependent; small Pd clusters might be imbedded in the support; and the Pd surface might not be completely covered with irreversibly chemisorbed hydrogen; etc. Our XAS data may shine some light to this issue.

Routine chemisorption measurements are made at $110^{\circ} \mathrm{C}$ to avoid the interference from the formation of palladium hydride[2]. We therefore conducted another experiment to study the status of hydrogen at $110^{\circ} \mathrm{C}$. We first introduced 1 atm of hydrogen to the hydrogen-free catalyst $\mathrm{B}$ at room temperature, then evacuated the catalyst at RT for 1 hour and $110^{\circ} \mathrm{C}$ for 0.5 hour. X-ray absorption data were collected at each step at room temperature. Results are shown in Table 2.

The Pd-Pd bond length changes from $2.750 \AA$ ( $0.032 \AA$ longer than that of the hydrogen-free particles) to $2.741 \AA$ $(0.010 \AA$ longer $)$, suggesting that surface coverage of the irreversibly chemisorbed hydrogen at $110^{\circ} \mathrm{C}$ is lower than that at RT. This may be one of the reasons why the chemisorption method underestimates the metal dispersion.

Table 2: Structure of Pd Clusters on Catalyst B Under Evacuation at Room Temperature and $110^{\circ} \mathrm{C}$

\begin{tabular}{|lll|}
\hline Conditions & Vac@RT & Vac@110 ${ }^{\circ} \mathrm{C}$ \\
$\mathrm{R} / \AA$ & $2.750 \pm 0.006$ & $2.741 \pm 0.007$ \\
$\mathrm{CN}$ & $4.0 \pm 0.5$ & $4.3 \pm 0.6$ \\
Sigma $^{2} / \AA^{2}$ & $0.0088 \pm 0.0007$ & $0.0102 \pm 0.0008$ \\
\hline
\end{tabular}

\section{Acknowledgments:}

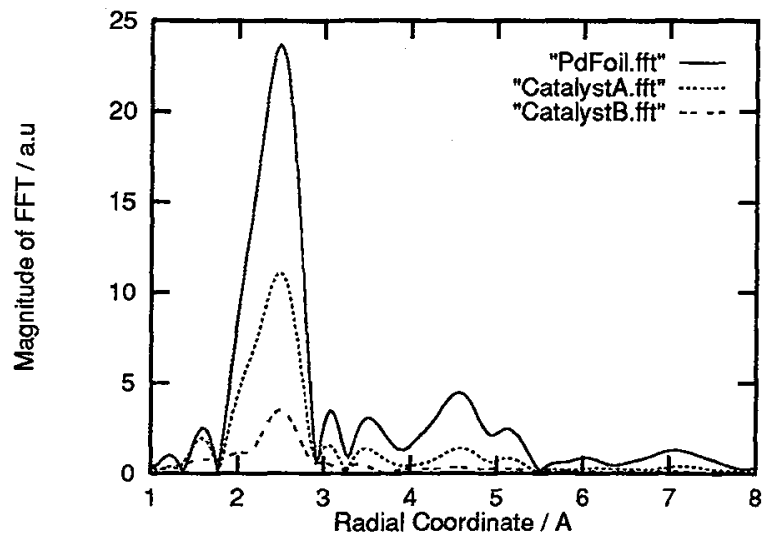

Figure 1: Fourier Transformed Fine Structure of Catalysts A and B

This work was carried out at the Brookhaven National Synchrotron Light Source, which is supported by the U. S. Department of Energy, Division of Materials Sciences and Division of Chemical Sciences.

\section{References}

[1] Stern E.A.,Newville M.,Ravel B.,Yacoby Y., and Haskel D., Physica B 208\&209(1995) 117-120.

[2] Benson J.E., Hwang H.S., and Boudart M., J. Catalysis 30 (1973) 146-153. 